\title{
SOME RECENT DEVELOPMENTS IN THE THEORY OF PARTIAL DIFFERENTIAL EQUATIONS
}

\author{
CHARLES B. MORREY, JR.
}

1. Introduction. When I sent in the title of this talk, I thought it appropriate for me, as the first speaker in this general session of the Society, to make some general remarks about the whole field of partial differential equations for the benefit of those who are not familiar with the field. I soon found out that a comprehensive survey of the field would not be possible in 35 minutes. A glance at a few sections in Mathematical Reviews on this subject will indicate why.

Therefore what I shall do in this lecture is to speak briefly on a few selected problems of general interest with which I have not had much direct contact and then give more and more details about those problems in elliptic differential equations and the calculus of variations with which I am more familiar. Thus, I shall say very little about hyperbolic, parabolic, and unclassified equations, other than to define them in the next section. I give the following justifications for these omissions: (a) lack of time, (b) the fact that many of the methods and ideas of functional analysis, etc., which I shall illustrate in connection with elliptic equations also apply to hyperbolic and parabolic ones, and (c) there are extensive treatments of hyperbolic equations by Leray [36] and Gårding [25] who are now jointly writing a book on that subject; also there is a self-contained set of lecture notes by Yosida [93] which exploits the many connections between parabolic equations and the theory of semi-groups.

During the past twelve years, there has been increasing activity in the writing of books and sets of notes on this subject, beginning with the 1950 book by I. G. Petrowsky, a translation of which was published in 1954 [72]. Since then the books by Miranda (elliptic type only) [42], Duff [20], Rosenbloom [75], and J. L. Lions [40] have appeared; that by Rosenbloom contains 734 references, about $1 / 3$ of which are to papers published between 1953 and 1957 ! Besides these, I know of books which are now being written by Hörmander, Browder, and myself which should appear shortly. No doubt there will be some small overlap between these books but, taken together, they should cover a large part of the field except possibly for applied mathematics and computation. It is regrettable that I shall not be

An address delivered before the Cincinnati meeting of the Society on January 23, 1962 by invitation of the Committee to Select Hour Speakers for Midwestern Meetings; received by the editors February 16, 1962. 
able to speak about these latter subjects, but I don't feel qualified; moreover, my very limited experience with the applied field suggests that interest in the applications is often a principal motivation for those working in it. I shall however speak later about some interesting mathematical applications. In the bibliography, I list a selection of sets of notes, proceedings of symposia, and expository articles; otherwise I list only papers referred to in the text and, of course, the bibliography is not complete.

2. Notation and terminology. For the most part, I shall use fairly standard notations: The boundary of a domain $G$ is denoted by $\partial G$. A function $u \in C_{\mu}^{m}(\bar{G}), 0<\mu \leqq 1$, iff $u$ can be extended to be of class $C^{m}$ on a domain $\supset \bar{G}$ and $u$ and all its derivatives of order $\leqq m$ satisfy a uniform Hölder condition with exponent $\mu$ (Lipschitz if $\mu=1$ ) on $\bar{G}$; $u \in C_{\mu c}^{m}(\bar{G})$ iff $u \in C_{\mu}^{m}(\bar{G})$ and the support of $u$ is in $G$. Diffeomorphisms (regular differentiable homeomorphisms) and domains of class $C_{\mu}^{m}$ are then defined as usual. All the definitions carry over to $C^{\infty}(\bar{G})$, etc. If $\phi$ is a function, $\nabla \phi$ denotes its gradient; if $\psi$ is a vector $|\psi|$ denotes its length; if $S$ is a set $|S|$ denotes its measure.

We shall be speaking of (partial) differential operators of the form

$$
L(x, D) u=\sum_{|\alpha| \S m} a_{\alpha}(x) D^{\alpha} u
$$

where

$$
\begin{aligned}
x=\left(x^{1}, \cdots, x^{\nu}\right), & \alpha=\left(\alpha_{1}, \cdots, \alpha_{\nu}\right) \\
\left(\alpha_{\epsilon} \geqq 0, \epsilon=1, \cdots, \nu\right) & \\
|\alpha|=\alpha_{1}+\cdots+\alpha_{\nu}, & D^{\alpha} u=D_{1}^{\alpha_{1}} \cdots D_{\nu}^{\alpha_{\nu}} u, \\
D_{\epsilon}^{\beta} u=\partial^{\beta} u /\left(\partial x^{\epsilon}\right)^{\beta}, & D^{\alpha} u=u \text { if }|\alpha|=0,
\end{aligned}
$$

the $\alpha_{\epsilon}$, of course, being integers $\geqq 0$. We usually assume that $x$ varies over a domain $G$ in the real $\nu$-space, but may allow $u$ and the $a_{\alpha}$ to be complex-valued. The principal part of the operator $L$ is the operator

$$
P L(x, D)=\sum_{|\alpha|=m} a_{\alpha}(x) D^{\alpha} .
$$

When speaking of systems of equations, (2.1) can be interpreted to stand for the vector operator

$$
\sum_{k=1}^{N} L_{j k}(x, D) u^{k} \quad\left(j=1, \cdots, N, u=u^{1}, \cdots, u^{N}\right)
$$

where the operators $L_{j k}$ may be of different orders; systems in which 
the order of $L_{j k}$ is $\leqq s_{j}+t_{k}$ have been studied by various authors $\left([65 ; 19 ; 58]\right.$ for example); if the $s_{j}$ are all 0 , the system is of order $t_{k}$ in $u^{k}$ in this case. In the case of a vector operator $L$, its principal part is the matrix $L_{0 j k}$ in which $L_{0 j k} \equiv 0$ or is the part of $L_{j k}$ which is of order exactly $s_{j}+t_{k}$.

We define the characteristic polynomial of the single operator $L$ to be

$$
\begin{aligned}
P L(x, \zeta) & =\sum_{|\alpha|=m} a_{\alpha}(x) \zeta^{\alpha} \quad\left(\zeta=\zeta_{1}, \cdots, \zeta_{\nu}\right), \\
\zeta^{\alpha} & =\zeta_{1}^{\alpha_{1}} \cdots \zeta_{\nu}^{\alpha_{\nu} .}
\end{aligned}
$$

For the vector operator (2.4), we define $P L(x, \zeta)$ to be the determinant of the polynomials $L_{0 j k}(x, \zeta)$. A hypersurface $s(x)=0$ is said to be characteristic at $x_{0}$ iff

$$
P L\left[x_{0}, \nabla s\left(x_{0}\right)\right]=0 .
$$

In the analytic case this corresponds to a situation in which the hypotheses of the corresponding Cauchy-Kovalewsky theorem are not satisfied. The operator (2.1) (or (2.4)) is said to be elliptic at $x_{0}$ iff $P L\left(x_{0}, \zeta\right) \neq 0$ for all real $\zeta \neq 0$. The single operator (2.1) is said to be hyperbolic (with respect to the first coordinate) for $x$ in a domain $G$ iff

$$
P L(x, \zeta)=a_{0}(x) \prod_{j=1}^{m}\left[\zeta_{1}-\lambda_{j}\left(x, \zeta_{1}^{\prime}\right)\right], \quad a_{0}(x) \neq 0,
$$

where the $\lambda_{j}$ are homogeneous of degree 1 in $\zeta_{1}^{\prime}=\left(\zeta_{2}, \cdots, \zeta_{v}\right)$ and are real and distinct if $\zeta_{1}^{\prime} \neq 0$. The operator (2.1) is parabolic (with respect to the first coordinate) iff

$$
L(x, D)=D_{1}-L_{1}\left(x, D_{2}, \cdots, D_{v}\right)
$$

where $L_{1}$ is elliptic in $D_{2}, \cdots, D_{p}$. In the cases of the hyperbolic and parabolic operators, the definitions could, of course, be generalized so as to include operators which assume their respective forms after an appropriate transformation of coordinates. The Laplace, wave, and heat equation operators

$$
\begin{array}{r}
D_{1}^{2}+D_{2}^{2}+D_{3}^{2}+D_{4}^{2}, D_{1}^{2}-D_{2}^{2}-D_{3}^{2}-D_{4}^{2} \text { and } D_{1}-D_{2}^{2}-D_{3}^{2}-D_{4}^{2} \\
(\nu=4)
\end{array}
$$

are well-known examples of operators of elliptic, hyperbolic, and parabolic types, respectively.

A unifying influence which has, at the same time, contributed greatly to the development of the subject is the increasing use of 
functional analysis, particularly the theories of Banach, Hilbert and other spaces, the Fourier transform, the Schwartz theory of distributions, and inequalities of Sobolev type. The use of the Fourier transform, together with a differential geometry technique involving a partition of unity, was at the root of Garding's now famous paper on the Dirichlet problem for higher order equations [24] (see also [23; $6 ; 90]$ ) which really started the very rapid advance of the last $10-12$ years.

There are certain Banach and Hilbert spaces of distributions, denoted by $H_{p}^{m}(G)$ (the Sobolev [83] spaces $W_{p}^{m}$ ), which have played a particularly important role in differential equations, potential theory and the calculus of variations. Actually Beppo Levi [37] started to study essentially these functions, where $m=1$ and $p=2$, in 1906 and essentially the same functions were studied by many other people over a long period of years (see [55] for some discussion and references). We shall illustrate the use of these functions in connection with elliptic equations and the calculus of variations but they have been used with great success by Leray and Gårding $[36 ; 25]$ in their work on hyperbolic equations and by Friedrichs in his interesting work on equations independent of type [22]. A distribution $u \in H_{p}^{m}(G)$ iff $u$ and its distribution derivatives of order $\leqq m$ correspond to actual functions in $L_{p}$; i.e., there exist functions $\phi_{\alpha} \in L_{p}(G)$, $0 \leqq|\alpha| \leqq m$, such that

$$
\begin{aligned}
\int_{G} \phi_{\alpha}(x) g(x) d x & =(-1)^{|\alpha|} \int_{G} \phi_{0}(x) D^{\alpha} g(x) d x & \\
& =(-1)^{|\alpha|} u\left(D^{\alpha} g\right), & g \in C_{c}^{\infty}(G) .
\end{aligned}
$$

If we define $\|u\|_{p}^{m} \geqq 0$ by

$$
\left(\|u\|_{p}^{m}\right)^{p}=\int_{G}\left[\sum_{0 \leq|\alpha| \S m} C_{\alpha}\left|\phi_{\alpha}(x)\right|^{2}\right]^{p / 2} d x
$$

for instance (there are many topologically equivalent norms), then $H_{p}^{m}(G)$ becomes a Banach space (here we can take $C_{\alpha}=|\alpha| ! /\left(\alpha_{1} !\right) \ldots$ $\left(\alpha_{\nu} !\right)$ if desired). The subspace $H_{p 0}^{m}(G)$ is the closure according to the norm $(2.10)$ of $C_{c}^{\infty}(\bar{G})$; it is known that $\|u\|_{p 0}^{m}$, defined by

$$
\left(\|u\|_{p 0}^{m}\right)^{p}=\int_{G}\left[\sum_{|\alpha|=m} C_{\alpha}\left|\phi_{\alpha}(x)\right|^{2}\right]^{p / 2} d x
$$

is equivalent to the norm (2.10) for $u \in H_{p 0}^{m}(G)$. The space $H_{p 0}^{m}(G)$ corresponds roughly to the vanishing of $u$ and its derivatives of order $\leqq m-1$ on $G$; no boundary term enters into the relation between the Fourier transform of $u$ and its derivatives $D_{\alpha} u$ with $0 \leqq|\alpha| \leqq m$. 
We sometimes say that $u$ has 0 Dirichlet data (of order $m$ ) on $\partial G$. In the case $p=2$, it is clear how to introduce inner products $(())$ and $(())_{0}$ in the respective spaces. The definition of the spaces $H_{p}^{m}(G)$ has been extended to cases where $G$ may be a manifold and $m$ may be any real number (see $[10 ; 5 ; 39 ; 40]$, etc.) and Lions [39] has been able to obtain very interesting results concerning the Navier-Stokes equations by using such spaces. And the spaces $H_{2}^{m}(G)$ with $m$ negative have been used to obtain easy proofs of the differentiability of the solutions of elliptic equations in the $C^{\infty}$ case (see [33 and 66]).

3. Some questions which are independent of type. It is not difficult to see that most differential equations of higher order and/or many variables are not of any of the three types: elliptic, hyperbolic, or parabolic. In this section we discuss briefly some questions which are independent of type.

A. The unique continuation theorem. In 1933, T. Carleman [12] announced (see also [13]) that any solution of an elliptic system of the first order of two equations for two functions of two variables which possesses a zero of infinite order vanishes identically. This result was recently extended by N. Aronszajn $[3 ; 3 \mathrm{a}]$ first to a single elliptic equation of the second order with real coefficients $\in C_{\mu}^{2}(G)$ and then to the second order systems arising from harmonic exterior differential forms [4]. Shortly afterwards and independently, H. O. Cordes [15] proved Aronszajn's first result in the case of $C^{2}$ coefficients. The results of Aronszajn and Cordes imply the uniqueness of the solution of the Cauchy problem (not the existence, of course) for the equations treated. The Cauchy problem for a single operator of order $m$ is that of showing the existence and/or uniqueness in the small of a solution of the given equation which assumes given "Cauchy data" (values of the function and first $m-1$ normal derivatives) on a given hypersurface. Calderón [9] has proved the uniqueness of the solution of the Cauchy problem with data on part of the hyperplane $\sigma \cdot x=c$ for arbitrary operators $L$ with real principal parts and no multiple characteristics in the sense that $P L(x, t \sigma+\zeta)$ has no multiple zeros in $t$ (real or complex) when $\zeta$ is real and $\neq 0$. For elliptic equations, Hörmander [29a] has recently improved Calderon's results. But counterexamples have been found by Myskis [60], Landis [32], Di Giorgi [17], Cohen [14], and Plis [73]. In these examples, either the coefficients were not in $C^{\infty}$ or the equations were not elliptic. Very recently, Plis [73a] and Cohen [14a] have found counterexamples where the equation is elliptic and the coefficients $\in C^{\infty}$. 
B. The nonexistence of solutions. In 1957, H. Lewy [38] published a simple example of a differential equation $L u=f$ ( $L$ analytic, $\left.f \in C^{\infty}\right)$ which has no solution in any neighborhood however small. On the other hand, any equation of this sort in which $L$ has constant coefficients always has solutions. Recently, Hörmander [29] has proved the following striking result: Let us write the operator $L$ in the form (2.1) where, however, $D_{\boldsymbol{\epsilon}}^{\beta} u$ means $(-i)^{\beta} \partial^{\beta} u /\left(\partial x^{c}\right)^{\beta}$, let $\bar{L}$ be the operator obtained by replacing the coefficients of $L$ (in this meaning of $D_{\epsilon}^{\beta}$ ) by their conjugates, and let $C_{2 m-1}$ be the part of order exactly $2 m-1$ in the commutator $\bar{L}_{0} L_{0}-L_{0} \bar{L}_{0}$, where $L_{0}=P L$, etc. Then (a) if $L u=f$ has a distribution solution on $G$ for each $f \in C_{c}^{\infty}(\bar{G})$, then $C(x, \xi)=0$ whenever $P L(x, \xi)=0, x \in G$, and $\xi \in R^{v}$ and (b) if this last condition does not hold on any nonvoid open subset $\omega$ of $G$, then there is an $f \in C^{\infty}(G)$, such that the $D^{\alpha} f(x) \rightarrow 0$ as $x \rightarrow \partial G$, for which there is no solution of $L u=f$ on any such $\omega$. In the same paper, he discusses some interesting cases in which solutions do exist.

C. Hypo-ellipticity. An operator $L$ with $C^{\infty}$ coefficients is said to be hypo-elliptic iff every solution $u$ of $L u=0 \in C^{\infty}(G)$. In case $L$ has constant coefficients, Hörmander [28] showed that a n.a.s.c. is that $L(\zeta) \rightarrow \infty$ uniformly as $\operatorname{Im} \zeta \rightarrow \infty$ with $\operatorname{Re} \zeta$ bounded; this class includes the elliptic and parabolic operators. Some interesting classes of hypo-elliptic operators with variable coefficients (including all elliptic ones) have been defined (see Nieto [63]) but their general characterization is still an open problem.

4. Elliptic equations and the calculus of variations. I shall begin by excluding a few subjects from the discussion. First of all, although the recent work on lower and upper bounds for eigenvalues is very important and includes much elegant and ingenious mathematics, I shall not speak of this subject except to mention the important work of Weinstein [91] the first part of which was published in 1937, the work on symmetrization published by Polya and Szegö [74] in 1945, and more recent papers by Payne, Weinberger, and others $([68 ; 70$; $67 ; 69$ ], etc.). I shall omit the interesting work of L. C. Young, W. H. Fleming and others (see [92], for instance) on generalized surfaces, since this work in the calculus of variations is too far removed from partial differential equations. I shall omit discussion of the work of Bers and Ahlfors on Riemann surfaces and the Morse theory [77a] for the same reason. There are other related topics such as pseudoanalytic functions, kernel functions, etc., on which books have already been written. Finally, I shall also omit potential theory since our third speaker will touch on this topic.

I shall now speak about the existence and differentiability theory 
for elliptic equations. I remark first that if $\nu>2$, the degree of the characteristic polynomial (for a linear elliptic equation or system) must be even, and if $\xi \perp \eta$, the equation $P L(x, \xi+z \eta)=0$ (of degree $2 m$ in $z$ ) has exactly $m$ roots $z$ with positive imaginary part; in this case we say that the equation or system is properly elliptic. If $\nu=2$, an elliptic equation need not be properly elliptic as the example

$$
\frac{\partial^{2} w}{\partial \bar{z}^{2}} \equiv \frac{1}{4}\left(\frac{\partial}{\partial x}+i \frac{\partial}{\partial y}\right)^{2} w=0
$$

shows; that equation has among its solutions all functions of the form $w=f(z)(z \bar{z}-1)$, where $f$ is analytic, all of which vanish on $x^{2}+y^{2}=1$.

I shall begin by sketching Gårding's solution [24] of the Dirichlet problem for a single equation

$$
\begin{aligned}
& \quad L u(x)+\lambda u(x)=f(x), \\
& x \text { on } G, D_{n}^{i} u(x)=0, x \text { on } \partial G, j=0, \cdots, m-1,
\end{aligned}
$$

of order $2 \mathrm{~m}$. Let us assume first of all, that $L=P L$, the $a_{\alpha}$ (see (2.1)) are real constants. Suppose $u$ is a solution, multiply (4.1) by $\bar{v} \in C_{c}^{\infty}(G)$ and integrate by parts in order to get rid of derivatives of $u$ higher than those of the $m$ th order. One obtains

$$
\begin{aligned}
& B(u, v)+\lambda(u, v)_{2}^{0}=L(v), \\
& B(u, v)=\int_{a} \sum_{\beta, \gamma} b_{\beta \gamma} D^{\beta} \bar{v} D^{\gamma} u d x, \quad L(v)=\int_{\theta}(-1)^{m} f \bar{v} d x .
\end{aligned}
$$

By approximations, (4.2) holds for all $v \in H_{20}^{m}(G)$. The integrations by parts are not unique, but if we take the Fourier transform of (4.2), we obtain

$$
B(u, v)=\int_{-\infty}^{\infty} \sum b_{\beta \gamma} y^{\beta} y^{\gamma} \hat{u}(y)(\hat{v}(y))-d y=\int_{-\infty}^{\infty} \sum a_{\alpha} y^{\alpha} \hat{u}(y)(\hat{v}(y))-d y .
$$

Obviously

$$
|B(u, v)| \leqq M_{1}\|u\|_{20}^{m} \cdot\|v\|_{20}^{m} .
$$

From the ellipticity, etc., we obtain

$$
\begin{aligned}
m_{1}|y|^{2 m} & \leqq \sum b_{\beta \gamma} y^{\beta} y^{\gamma} \equiv \sum a_{\alpha} y^{\alpha} \leqq M|y|^{2 m}, \\
\int_{G}\left|\nabla^{m} u\right|^{2} d x & =\int_{-\infty}^{\infty}|y|^{2 m}|a(y)|^{2} d y .
\end{aligned}
$$

From this, it follows that 


$$
B(u, u) \geqq m_{1}\|u\|^{2}, \quad\|u\|=\|u\|_{20}^{m} .
$$

The fact (4.6) is not at all evident from (4.2) since the ellipticity condition (if $m>1$ ) in (4.5) does not imply that the integrand in (4.2) with $v=u$ is positive definite. The fact emerged clearly only because of the use of the Fourier transform. Actually Van Hove [89] had obtained a corresponding result for second order systems.

In case the coefficients $b_{\beta \gamma}$ in (4.2) are variable but real and lower order terms are allowed, (4.4) still holds; if the $b_{\beta \gamma}$ with $|\beta|=|\gamma|=m$ are continuous, (4.6) can be replaced by

$$
B(u, u) \geqq\left(m_{1}-\epsilon\right)\left(\|u\|_{2,0}^{m}\right)^{2}-C(\epsilon)\left(\|u\|_{2}^{0}\right)^{2}
$$

which is known as Garding's inequality [24]. It is proved by using the results above and a partition of unity. By Hilbert space theory, it follows that there are transformations $T$ and $U$ and an element $w \in H_{20}^{m}(G)$ such that

$$
\begin{aligned}
B(u, v) & =((T u, v))_{20}^{m}, \quad \int_{G} u \bar{v} d x=((U u, v))_{20}^{m}, \\
L(v) & =((w, v))_{20}^{m} ;
\end{aligned}
$$

$T$ is bounded (from (4.4)) and it follows from compactness theorems for $H_{20}^{m}$ functions that $U$ is completely continuous. From (4.7), it follows that there is a real $\lambda_{0}$ so large that

$$
\left(\left(T u+\lambda_{0} U u, u\right)\right)_{20}^{m}=B(u, u)+\lambda_{0}\left(\|u\|_{2}^{0}\right)^{2} \geqq \frac{1}{2} m_{1}\left(\|u\|_{20}^{m}\right)^{2}
$$

for all $u$. It follows from the Lemma of Lax and Milgram [33a] that the operator $T+\lambda U$ is invertible for all except an isolated set of eigenvalues of $\lambda$.

Now, if the coefficients in $L$ are variable and lower order terms are present, (4.1) cannot be reduced to (4.2) unless the coefficients $a_{\alpha}$ with $|\alpha|>m \in C_{1}^{|\alpha|-m-1}(\bar{G})$ at least. Then (4.1) can be reduced to (4.2) by integration by parts in such a way that the $b_{\beta \gamma} \in C_{1}^{|\beta|-1}$ for $|\beta|>0$. The idea of the existence theory is to replace (4.1) by (4.2), which is, in turn equivalent to the equation

$$
T u+\lambda U u=w .
$$

The results of the last paragraph show that (4.9) is solvable according to the usual Fredholm alternative. Let us pick a $\lambda$ for which this is solvable and absorb the term $\lambda U u$ into $T u$. The solution $u \in H_{20}^{m}(G)$, whereas it is desired for $u \in H_{2}^{2 m}(G)$. To show that $u$ has more deriva- 
tives, we proceed as follows: If certain coefficients are differentiable, we may choose a $v \in C_{c}^{\infty}(G)$, replace $v$ by $h^{-1}\left[v\left(x-h e_{\gamma}\right)-v(x)\right]$ (e being a unit vector in the $x^{\gamma}$ direction, $\left.\gamma=1, \cdots, \nu\right)$, and change variables to eliminate terms in $v\left(x-h e_{\gamma}\right)$; the result is that

$$
u_{h}(x)=h^{-1}\left[u\left(x+h e_{\gamma}\right)-u(x)\right]
$$

satisfies an equation like (4.2) (with $G$ replaced by $D^{\prime}$ with $\bar{D}^{\prime} \subset G$ ) where the right side also contains terms $\sum b_{\alpha}\left(D^{\alpha} v\right)$ - where $|\alpha|=1$; it is not necessary that $f$ be differentiable, but if not, it contributes to one of these latter terms. By replacing $v$ again by $\zeta^{2 m} u_{h}$ and setting $U=\zeta^{m} u_{h}$ where $\zeta \in C_{c}^{\infty}(G)$ and $\zeta(x) \equiv 1$ on $D\left(\bar{D} \subset D^{\prime}\right)$ one obtains, by using bounds derivable from (4.2), (4.5), and (4.6) on the new equations that the $\left\|u_{h}\right\|_{2, D}^{m}$ are uniformly bounded and that one can let $h \rightarrow 0$ and obtain an equation like (4.2) for the derivative $D_{\gamma} u$, which holds on any $D$ with $\bar{D} \subset G$. This may be continued as long as the coefficients have the requisite derivatives; if they are of class $C^{\infty}$, it follows from a Sobolev lemma that $u \in C^{\infty}$. In case the $b_{\beta \gamma}$ $\in C_{1}^{|\beta|-1}(|\beta|>0), u \in H_{2}^{2 m}(D)$ on any $D$ with $\bar{D} \subset G$. If $G$ is of class $C^{2 m}$, a boundary neighborhood can be mapped regularly so a part of $\partial G$ corresponds to a part of $x^{\nu}=0$; the difference quotient procedure above can then be applied in the tangential directions. Thus (by using some additional tricks) it can be shown that $u \in H_{2}^{2 m}(G)$. By using the well-known results of Calderón-Zygmund [11], it can be shown that $u \in H_{p}^{2 m}(G)$ for any $p$. If $G \in C_{\mu}^{2 m}$ and the $a_{\alpha} \in C_{\mu}^{|\alpha|-m}(\bar{G})$, then $u \in C_{\mu}^{2 m}(\bar{G})$.

The procedure in the preceding paragraph clearly requires that the $a_{\alpha}$ with $|\alpha|>m$ be differentiable $|\alpha|-m$ times, whereas in the case of a single second order equation no such restriction is necessary. In June of 1958 [52], I announced that I was able to remove the restrictions. I discussed this with Browder and Nirenberg who each supplied a proof after some thought; Nirenberg's proof is to be found in [2, pp. 693-695] and Browder's is in [7a]. The result extends to the case of the Dirichlet problem for the so-called "strongly elliptic" systems (see [65]).

More general boundary value problems of the form

$$
L u=f \text { in } G, \quad B_{j} u=0 \text { on } \partial G, \quad j=1, \cdots, m,
$$

where $L$ is properly elliptic with continuous leading coefficients and the $B_{j}$ are operators of order $r_{j}<2 m$ (sufficiently smooth-details omitted here) have been studied. If $G$ is bounded and of class $C^{2 m}$ and the $B_{j}$ and $L$ satisfy a certain "complementing condition" of an algebraic nature (see, for example, $[7$ or 2, p. 626$]$ ), on $\partial G$, then it 
has been shown essentially simultaneously by Agmon, Browder, Douglis, and Nirenberg that any $u \in H_{p}^{2 m}(G)$ which satisfies the boundary conditions in (4.10) satisfies

$$
\|u\|_{p, G}^{2 m} \leqq C\left(\|L u\|_{p, G}^{0}+\|u\|_{1, G)}^{0}\right) .
$$

If $G$ is of class $C_{\mu}^{2 m}$ and the corresponding Hölder continuity requirements $(0<\mu<1)$ are put on the coefficients, then any $u \in C_{\mu}^{2 m}(G)$ which satisfies the boundary conditions in (4.10) satisfies

$$
\|\| u \|\left.\right|_{\mu, G} ^{2 m} \leqq C\left(\|\mid L u\|\left\|_{\mu, G}^{0}+\right\| u \|_{1, G}^{0}\right) .
$$

These a priori bounds show that, for each $\lambda$, the null space of the operator $L+\lambda I$ (assuming $B_{j} u=0$ ) is finite dimensional. However, for the general $L$ and $B_{j}$, it is not known whether there is any value at all of $\lambda$ for which $L+\lambda I$ is invertible (with those boundary conditions). In order to get around this difficulty, Schechter $[76 ; 77]$ and Browder [7] have considered an adjoint problem with adjoint boundary conditions and have found that the given problem is solvable iff the null space of the adjoint problem is 0-dimensional. Schechter [77] and Browder [7] have considered other kinds of boundary conditions including some mixed ones. Browder [7] has carried over the estimates above for certain unbounded domains and has obtained some extensive corresponding results for parabolic equations. Agmon [1] has proved a generalized maximum principle for the solutions of elliptic equations which extends the one of Miranda [43] to the Dirichlet problem for the general elliptic operator.

Progress in nonlinear elliptic equations has been very slow. In 1938-1939, Leray published two very interesting papers $[34 ; 35]$ on single nonlinear equations in two variables. In 1953, Nirenberg [64] proved the existence of solutions of quasi-linear differential equations in two variables of the form

$$
a\left(x, y, z, z_{x}, z_{y}\right) z_{x x}+2 b z_{x y}+c z_{y y}=0
$$

where the coefficients are bounded and satisfy

$$
a \xi^{2}+2 b \xi \eta+c \eta^{2} \geqq m_{1}\left(\xi^{2}+\eta^{2}\right), \quad m_{1}>0 .
$$

Some very interesting results concerning minimal surfaces and equations of minimal surface type in two dimensions have been obtained by Finn $[20 a ; 20 b]$, Bers [5a], H. Jenkins [29b], and others. Additional differentiability of the solutions of certain nonlinear equations and systems in more variables has been proved by Nirenberg [19] and myself $[44 ; 49]$. And Petrowsky [71], A. Friedman [21], and I [51] have given proofs of S. Bernstein's theorem on the analyticity 
of the solutions of analytic elliptic systems, the latter two proving analyticity at an analytic boundary for strongly elliptic systems. The proof of Petrowsky is long, but those of Friedman and Morrey are comparatively short. The results of Agmon, Douglis, and Nirenberg [2] include a perturbation theorem for a nonlinear equation or system and some results of Cordes [16] lead to a proof of the existence of the solution of a single quasi-linear equation of the form

$$
\sum a^{\alpha \beta}(x, z, \nabla z) z,{ }_{\alpha \beta}(x)=0
$$

with given smooth boundary values on a smooth boundary provided that the eigenvalues $\lambda_{1}, \cdots, \lambda_{\nu}$ of the $a^{\alpha \beta}$ matrix in (4.13) satisfy his $K_{\epsilon}^{\prime}$ condition uniformly. D. Gilbarg has recently obtained an existence theorem (unpublished) for certain quasi-linear equations. Extensive results concerning such equations "in divergence form" have been obtained by Ladyženskaya and Ural'tseva $[31 ; 31 \mathrm{a}]$.

Otherwise, the variational method has successfully attacked many nonlinear equations and systems which are the Euler equations of variational problems. Many years ago $[45 ; 46]$, I extended the existence theory of Tonelli (see $[86 ; 87 ; 88]$, for instance) to the case of nonparametric integrals of the form

$$
I(z, G)=\int_{G} f(x, z, \nabla z) d x, \quad x=\left(x^{1}, \cdots, x^{\nu}\right), \quad z=\left(z^{1}, \cdots, z^{N}\right)
$$

where $f(x, z, p)\left(p=p_{\alpha}^{i}, \alpha=1, \cdots, \nu, i=1, \cdots, N\right)$ is continuous in $(x, z, p)$ and convex in $p$ for each $(x, z)$ with

$$
f(x, z, p) \geqq f_{0}(p), \quad \lim _{|p| \rightarrow \infty}|p|^{-1} f_{0}(p)=+\infty ;
$$

the existence theorems included some allowing variable boundary values. If $N=1$, it is well known that a minimizing function may not exist if $f$ is not convex in $p$. For $N>1$, the situation is more complicated; I have investigated the more general integrals [48], but the results are not completely satisfactory. Serrin $[79 ; 78 ; 79 a]$ has extended my results for the case $N=1$. Gel'man [26] has generalized some of these results to variational problems of higher order.

At about the same time, I $[45 ; 46]$ proved that any locally minimizing extremal of such an integral $\in C_{\mu}^{1}(D)$, in fact $C_{\mu}^{n}(D)$ if $f \in C_{\mu}^{n}(n \geqq 2)$, for each $D$ with $\bar{D} \subset G$, provided that $f$ satisfies the conditions (4.17) below with $k=1$ and $\nu=2$. I used those results to prove the differentiability of my in-the-large solutions of the problem of Plateau on a Riemannian manifold [47]. Some of these results were generalized somewhat by Sigalov $[80 ; 81]$ and Silova [82] with $\nu$ still equal to 2 . 
The methods used above in the case $\nu=2$ would not generalize and it was not until after the now famous results of Di Giorgi [18] and Nash [61], as simplified still further by Moser [59] that the differentiability of the solutions of any nonquadratic variational problems with $\nu>2$ were proved. The Di Giorgi-Nash results concern equations of the form (4.2) where $N=1, m=1$, the $b_{\beta \gamma}$ are merely bounded and measurable, $f=0$, and $b_{\beta \gamma}=0$ if either $\beta$ or $\gamma=0$. Between 1958 and 1960, Stampacchia $[84 ; 85]$ and I [54] generalized the Di Giorgi-Nash results to equations (4.2) in which lower-order and nonhomogeneous terms are allowed; such equations arise in the differentiability theory for the solutions of variational problems.

Ladyženskaya and Ural'tseva $[31 ; 31 a]$ have shown that any bounded solution $z$ of a variational problem in which $f$ satisfies the conditions (4.17) below (essentially) with any $k>1 / 2$ is of class $C_{\mu}^{n}(D)$ for each $D \subset G$ provided that $f \in C_{\mu}^{n}$ for some $n \geqq 3$. Stampacchia has recently obtained a simple result concerning variational problems in which $f=f(p)$ and $G$ is strictly convex. In the fall of 1959 a student, E. R. Buley, working under my direction, had obtained some results for integrals in which $f$ satisfies the conditions $(4.16)^{\prime}$ below. In January, 1960, J. Moser kindly communicated his simplification of the Di Giorgi-Nash results. This enabled us to obtain the results stated below. These results are closely related to those of Ladyženskaya and Ural'tseva but the methods appear to be entirely different.

The conditions on $f$ are as follows: $f \in C_{\mu}^{n}, n \geqq 2,0<\mu<1, N=1$, and $f$ is to satisfy some one of the following sets of hypotheses for all $(x, z, p)$ :

$$
\begin{aligned}
& m_{1} V^{k}-K \leqq f(x, z, p) \leqq M_{1} V^{k}, 0<m_{1} \leqq M_{1}, k>1 / 2 \\
& \sum\left(f_{p_{\alpha}}^{2}+f_{p_{\alpha} x \gamma}^{2}+f_{z}^{2}+f_{z x \gamma}^{2}\right) \leqq M_{1} V^{2 k-1} \\
& \sum\left(f_{p_{\alpha} z}^{2}+f_{z z}^{2}\right) \leqq M_{1} V^{2 k-2} \\
& \quad\left(V=1+|z|^{2}+|p|^{2}\right), \\
& m_{1} V^{k-1}|\lambda|^{2} \leqq f_{p_{\alpha} p_{\beta}} \lambda_{\alpha} \lambda_{\beta} \leqq M_{1} V^{k-1}|\lambda|^{2}, \\
& \text { same as }(4.16) \text { with } f=f(x, p), V \\
& m_{1} V^{k}-K \leqq f(x, z, p) \leqq M_{1} V^{k}, \quad 0<m_{1} \leqq M_{1}, k \geqq \nu / 2 \\
& \sum\left(f_{z}^{2}+f_{z z}^{2}+f_{z x \gamma}^{2}\right) \leqq M_{1} V^{2 k}, \\
& \sum\left(f_{p_{\alpha}}^{2}+f_{p_{\alpha} z}^{2}+f_{p_{\alpha} x \gamma}^{2} \leqq M_{1} V^{2 k-1},\right. \\
& m_{1} V^{k-1}|\lambda|^{2} \leqq f_{p_{\alpha} p_{\beta}}^{m} \lambda_{\alpha} \lambda_{\beta} \leqq M_{1} V^{k-1}|\lambda|^{2}
\end{aligned}
$$


In the case of (4.16) or $(4.16)^{\prime}$ with $k \geqq 1$, any extremal $z \in C_{\mu}^{n}(G)$. $z$ is an extremal for the integral (4.14) iff it satisfies

$$
\int_{G}\left[\zeta, \alpha f_{p_{\alpha}}(x, z, \nabla z)+\zeta f_{z}(x, z, \nabla) z\right] d x=0, \quad \zeta \in C_{c}^{1}(G) ;
$$

any minimizing function is an extremal under any of the hypotheses above on $f$. In the cases (4.16) or (4.16)' with $1>k>1 / 2$, or (4.17) with $k \geqq \nu / 2$, we have the following result: if $z^{*} \in H_{2 k}^{1}(G)$ and $G$ is bounded, there exists a minimizing extremal $z \in C_{\mu}^{n}(G)$ such that $z-z^{*}$ $\in H_{2 k, 0}^{1}(G)$.

To see the difference between the conditions (4.16) and (4.17), we note that

$$
f(x, z, p)=a^{\alpha \beta}(x, z) p_{\alpha} p_{\beta}+2 b^{\alpha}(x, z) p_{\alpha}+c(x, z)
$$

satisfies (4.17) with $k=1$, if $a^{\alpha \beta} p_{\alpha} p_{\beta}$ is positive definite, etc., but does not satisfy (4.16).

One annoying feature of these results is that $N$ is restricted to be 1 . This rules out most applications to differential geometry. The removal of this restriction appears to await the extension of the Di Giorgi-Nash-Moser results to the case of vector functions.

In conclusion, I would like to mention a few problems in which the methods of differential equations or the calculus of variations have led to a solution.

(i) Harmonic integrals. The variational technique was very efficient in the study of harmonic integrals undertaken with Eells $[57 ; 50]$. One of the boundary problems studied in part II is not normal since the null space is not finite dimensional.

(ii) Almost complex manifolds. Newlander and Nirenberg [62] proved by methods of differential equations that an almost complex manifold can be given a complex analytic structure.

(iii) The analytic embedding of an abstract real-analytic manifold. The possibility of such an embedding for compact manifolds with given analytic metric was first proved by Bochner [8] in 1937. This result was generalized to noncompact manifolds with countable topology by Malgrange in 1957 [41]. In 1958, I [53] proved Bochner's result without assuming the existence of an analytic metric. This result was generalized to noncompact, etc., manifolds by Grauert [27], using complex variable methods. My proof involved the solution of a $\bar{\partial}$-Neumann problem for complex forms of degrees 0 and 1 on certain complex manifolds; part of those results have recently been generalized by J. J. Kohn [30]. This leads to another nonregular boundary problem in differential equations of which the null space is not finite dimensional. 
It is to be hoped that these methods will prove useful in solving interesting problems in differential geometry and other branches of mathematics. However, it appears necessary to make a great deal of progress in both differential equations and the calculus of variations before they can be used extensively as tools in these other branches.

\section{BIBLIOGRAPHY}

1. S. Agmon, Maximum theorems for solutions of higher order elliptic equations, Bull. Amer. Math. Soc. 66 (1960), 77-80.

2. S. Agmon, A Douglis and L. Nirenberg, Estimates near the boundary for solutions of elliptic partial differential equations satisfying general boundary conditions. I, Comm. Pure Appl. Math. 12 (1959), 623-727.

3. N. Aronszajn, Sur l'unicitê du prolongement des solutions des équations aux dérivées partielles elliptiques du second ordre, C. R. Acad. Sci. Paris 242 (1956), 723-725.

3a. - A Anique continuation theorem for solutions of elliptic partial differential equations or inequalities of second order, J. Math. Pures. Appl. 36 (1957), 235-249.

4. N. Aronszajn, A. Krzywicki and J. Szarski, Unique continuation theorem for exterior differential forms on Riemannian manifolds, ONR Tech. Rep. No. 25, Univ. of Kansas, Lawrence, Kans., 1960; to appear in Arkiv. for Math.

5. N. Aronzajn, Associated spaces, interpolation theorems and the regularity of the solutions of differential problems, Proc. Sympos. Pure Math. Vol. 4, pp. 23-32, Amer. Math. Soc., Providence, R. I., 1962.

5a. L. Bers, Non-linear elliptic equations without non-linear entire solutions, J. Rational Mech. Anal. 3 (1954), 767-787.

6. F. E. Browder, The Dirichlet problem for linear elliptic equations of arbitrary even order with variable coefficients, Proc. Nat. Acad. Sci. U.S.A. 38 (1952), 230-235.

7. - A priori estimates for elliptic and parabolic equations, Proc. Sympos. Pure Math. Vol. 4, pp. 73-81, Amer. Math. Soc., Providence, R. I., 1962.

7a - On the spectral theory of elliptic differential operators. I, Math. Ann. 142 (1961), 22-130.

8. S. Bochner, Analytic mapping of compact Riemann spaces into Euclidean space, Duke Math. J. 3 (1937), 339-354.

9. A. P. Calderón, Uniqueness in the Cauchy problem for partial differential equations, Amer. J. Math. 80 (1958), 16-36.

10. - Lebesgue spaces of differentiable functions, Proc. Sympos. Pure Math. Vol. 4, pp. 33-49, Amer. Math. Soc., Providence, R. I., 1962.

11. A. P. Calderón and A. Zygmund, Singular integral operators and differential equations, Amer. J. Math. 79 (1957), 901-921.

12. T. Carleman, Sur les systèmes linéaires aux dérivées partielles $d u$ premier ordre à deux variables, C.R. Acad. Sci. Paris 197 (1933), 471-474.

13. - Sur un problème d'unicité pour les systèmes d'équations aux dérivées partielles à deux variables indépendentes, Archiv. f. Mat. Fys. o Astr. (26B) 17 (1939), 1-9.

14. P. J. Cohen, The non-uniqueness of the Cauchy problem, Tech. Rept. No. 93 (Dec. 30, 1960), Appl. Math. and Stat. Lab., Stanford University.

14a. - (to appear).

15. H. O. Cordes, Ueber die Bestimmtheit der Lösungen elliptischer Differentialgleichungen durch Anfangsvorgaben, Nachr. Akad. Wiss. Göttingen, Math.-Phys. K1. (IIa) No. 11 (1956), 239-253. 
16. - Zero order a priori estimates for solutions of elliptic differential equations, Proc. Sympos. Pure Math. Vol. 4, pp. 157-166, Amer. Math. Soc., Providence, R. I., 1962.

17. E. Di Giorgi, Un esempio di non-unicità della soluzione del problema di Cauchy, relativo ad una equazione differenziale lineare a derivate parziali di tipo parabolico, Rend. Mat. e Appl. (5) 14 (1955), 382-387.

18. - Sulla differenziabilitd e l'analyticitd delle estremali degli integrali multipli regolari, Mem. Accad. Sci. Torino Cl. Sci. Fis. Mat. Nat. (3) 3 (1957), 25-43.

19. A. Douglis and L. Nirenberg, Interior estimates for elliptic systems of partial differential equations, Comm. Pure Appl. Math. 8 (1955), 503-538.

20. G. F. D. Duff, Partial differential equations, Math. Expositions No. 9, Toronto Univ. Press, Toronto, 1956.

20a. R. Finn, On equations of minimal surface type, Ann. of Math. (2) 60 (1954), 367-416.

20b. - On a problem of type with application to elliptic partial differential equations, J. Rational Mech. Anal. 3 (1954), 789-799.

21. A. Friedman, On the regularity of the solutions of non-linear elliptic and parabolic systems of partial differential equations, J. Math. Mech. 7 (1958), 43-59.

22. K. O. Friedrichs, Symmetric positive linear differential equations, Comm. Pure Appl. Math. 11 (1958), 333-418.

23. L. Gårding, Le problème de Dirichlet pour les équations aux dérivées partielles elliptiques linéaires dans les domaines bornés, C. R. Acad. Sci. Paris 233 (1951), 15541556.

24. - Dirichlet's problem for linear elliptic partial differential equations, Math. Scand. 1 (1953), 55-72.

25. - Cauchy's problem for hyperbolic equations, Dept. of Math., Univ. of Chicago, Chicago, Ill., 1958.

26. I. V. Gel'man, The minimum problem for a non-linear functional, Leningrad. Gos. Ped. Inst. Uč. Zap. 166 (1958), 255-263.

27. H. Grauert, On Levi's problem and the imbedding of real-analytic manifolds, Ann. of Math. (2) 68 (1958), 460-472.

28. L. Hörmander, On the theory of general partial differential operators, Acta Math. 94 (1955), 160-248. 173.

29. - Differential equations without solutions, Math. Ann. 140 (1960), 169-

29a. - On the uniqueness of the Cauchy problem. II, Math. Scand. 7 (1959), $177-190$.

29b. H. Jenkins, On quasi-linear elliptic equations which arise from variational problems, J. Math. Mech. 10 (1961), 705-727.

30. J. J. Kohn, Solution of the $\bar{\partial}$-Neumann problem on strongly pseudo-convex manifolds, Proc. Nat. Acad. Sci. U.S.A. 47 (1961), 1198-1202.

31. O. A. Ladyženskaya and N. N. Uralt'seva, On the smoothness of weak solutions of quasi-linear equations in several variables and of variational problems, Comm. Pure Appl. Math. 14 (1961), 481-495.

31a. - Quasi-linear elliptic equations and variational problems with many independent variables, Russian Math. Surveys, London Math. Soc. (16) 1 (1961), 17-91.

32. E. M. Landis, An example of non-uniqueness of solutions of Cauchy's problem for a system of the form $\partial u_{i} / \partial t=\sum_{j} A_{i j}\left(\partial u_{j} / \partial x\right)+\sum_{j} B_{i j} u_{j}+f_{i}(i, j=1,2)$, Mat. Sb. (N.S.) 27(69) (1950), 319-323. 
33. P. Lax, On Cauchy's problem for hyperbolic equations and the differentiability of the solutions of elliptic equations, Comm. Pure Appl. Math. 8 (1955), 615-633.

33a. P. D. Lax and A. N. Milgram, Parabolic equations, Ann. of Math. Studies No. 33, Princeton Univ. Press, Princeton, N. J., 1954; pp. 167-190, especially p. 169.

34. J. Leray, Majoration des dérivées secondes des solutions d'un problème de Dirichlet, J. Math. Pures Appl. (9) 17 (1938), 89-104.

35. — Discussion d'un problème de Dirichlet, J. Math. Pures Appl. (9) 18 (1939), 249-284.

36. - Lectures on hyperbolic equations with variable coefficients, Institute for Advanced Studies, Princeton, N. J., 1952.

37. B. Levi, Sul principio di Dirichlet, Rend. Circ. Nat. Palermo 22 (1906), 293359.

38. H. Lewy, An example of a smooth linear partial differential equation without solution, Ann. of Math. (2) 66 (1957), 155-158.

39. J. L. Lions, Sur certains problèmes différentiels non linéaires, C. R. Acad. Sci. Paris 252 (1961), 657-659.

40. - Equations différentielles opérationnelles et problèmes aux limites, Die Grundlehren der mathematischen Wissenschaften, Springer, Berlin, 1961.

41. B. Malgrange, Plongement des variêtés analytiques réelles, Bull. Soc. Math. France 85 (1957), 101-113.

42. C. Miranda, Equazioni alle derivate parziali di tipo ellittico, Ergebnisse der Mathematik und ihrer Grenzgebiete (N.F.), Heft 2, Springer, Berlin, 1955.

43. - - Teorema del massimo modulo e teorema di esistenza e di unicitd per il problema di Dirichlet relativo alle equazioni ellittiche in due variabili, Ann. Mat. Pura Appl. 46 (1958), 265-311.

44. C. B. Morrey, Jr., On the solutions of quasi-linear elliptic partial differential equations, Trans. Amer. Math. Soc. 43 (1938), 126-166.

45. - Existence and differentiability theorems for the solutions of variational problems, Bull. Amer. Math. Soc. 46 (1940), 439-458.

46. - Multiple integral problems in the calculus of variations and related topics, Univ. California Publ. Math. (N.S.) 1 (1943), 1-130.

47. - The problem of Plateau on a Riemannian manifold, Ann. of Math. (2) 49 (1948), 807-851.

48. - Quasi-convexity and the lower semicontinuity of multiple integrals, Pacific J. Math. 2 (1952), 25-53.

49. - Second order elliptic systems of differential equations, Ann. of Math. Studies No. 33, Princeton Univ. Press, Princeton, N. J., 1954; pp. 101-159.

50. - A variational method in the theory of harmonic integrals. II, Amer. J. Math. 78 (1956), 137-170.

51. - On the analyticity of the solutions of analytic non-linear elliptic systems of partial differential equations. I, II, Amer. J. Math. 80 (1958), 198-237.

52. - Elliptic differential equations with Hölder continuous coefficients, Amer. Math. Soc. Notices 5 (1958), 466.

53. - The analytic embedding of abstract real-analytic manifolds, Ann. of Math. (2) 68 (1958), 159-201.

54. - Second order elliptic equations in several variables and Hölder continuity, Math. Z. 72 (1959), 146-164.

55. - Multiple integral problems in the calculus of variations and related topics, Ann. Scuola Norm. Pisa (III) 14 (1960), 1-61.

56. - Existence and differentiability theorems for variational problems for 
multiple integrals, Partial differential equations and continuum mechanics, Univ. of Wisconsin Press, Madison, Wis., 1961.

57. C. B. Morrey, Jr. and J. Eells, Jr., A variational method in the theory of harmonic integrals. I, Ann. of Math. (2) 63 (1956), 91-128.

58. C. B. Morrey, Jr. and L. Nirenberg, On the analyticity of the solutions of linear elliptic systems of partial differential equations, Comm. Pure Appl. Math. 10 (1957), 271-290.

59. L. Moser, A new proof of di Giorgi's theorem concerning the regularity problem for elliptic differential equations, Comm. Pure Appl. Math. 13 (1960), 457-468.

60. A. Myskis, On the method of A. Haar in a question of the uniqueness of the solution of the problem of Cauchy for a system of partial differential equations, Dokl. Akad. Nauk. SSSR (N.S.) 58 (1947), 21-24.

61. J. Nash, Continuity of the solutions of parabolic and elliptic equations, Amer. J. Math. 80 (1958), 931-954.

62. A. Newlander and L. Nirenberg, Complex analytic coordinates in almost complex manifolds, Ann. of Math. (2) 65 (1957), 391-404.

63. J. Nieto, Eine Charakterisierung der elliptischen Differentialoperatoren, Math. Ann. 141 (1960), 22-42.

64. L. Nirenberg, On non-linear elliptic partial differential equations and Hölder continuity, Comm. Pure Appl. Math. 6 (1953), 103-156.

65. - Remarks on strongly elliptic partial differential equations, Comm. Pure Appl. Math. 8 (1955), 648-674.

66. - On elliptic partial differential equations, Ann. Scuola Norm. Pisa (III) 13 (1959), 1-48.

67. L. E. Payne, Inequalities for eigenvalues of membranes and plates, J. Rational Mech. Anal. 4 (1955), 517-529.

68. L. E. Payne and H. F. Weinberger, New bounds in harmonic and biharmonic problems, J. Math. Phys. 33 (1955), 291-307.

69. - Lower bounds for frequencies of elastically supported membranes and plates, J. Soc. Indust. Appl. Math. 5 (1957), 171-182.

70. L. E. Payne and A. Weinstein, Capacity, virtual mass, and generalized symmetrization, Pacific J. Math. 2 (1952), 633-641.

71. I. Petrovsky, Sur l'analyticitê des solutions des systèmes d'équations différentielles, Rec. Mat. N.S. Mat. Sbornik 5(47) (1939), 3-70.

72. - Lectures on partial differential equations. I (Translation from the 1950 Russian edition), Interscience, New York, 1954.

73. A. Plis, The problem of uniqueness for the solution of a system of partial differential equations, Bull. Acad. Polon. Sci. Cl. III 2 (1954), 55-57.

73a. - A smooth linear elliptic differential equation without any solution in a sphere, Comm. Pure Appl. Math. 14 (1961), 599-617.

74. G. Polya and G. Szegö, Inequalities for the capacity of a condenser, Amer. J. Math. 67 (1945), 1-32.

75. P. C. Rosenbloom, Linear partial differential equations, Surveys in Applied Math., Vol. 5, New York, 1958.

76. M. Schechter, General boundary value problems for elliptic partial differential equations, Comm. Pure Appl. Math. 12 (1959), 457-486.

77. - Various types of boundary conditions for elliptic equations, Comm. Pure Appl. Math. 13 (1960), 407-425.

77a. H. Seifert and W. Threlfall, Variationsrechnung im Grossen (Theorie von Marston Morse), Chelsea, New York, 1951. 
78. J. Serrin, On a fundamental theorem of the calculus of variations. A new definition of the integral for non-parametric problems in the calculus of variations, Acta Math. 102 (1959), 1-32.

79. - Dirichlet's principle in the calculus of variations, Proc. Sympos. Pure Math. Vol. 4, pp. 17-22, Amer. Math. Soc., Providence, R. I., 1962.

79a. - On the definition and properties of certain variational integrals, Trans. Amer. Math. Soc. 101 (1961), 139-167.

80. A. G. Sigalov, Two dimensional problems in the calculus of variations, Uspehi Mat. Nauk (N.S.) 6 (1951), 16-101; Amer. Math. Soc. Transl. No. 83 (1953).

81. - Two dimensional problems of the calculus of variations in non-parametric form, Trudy Moskov. Mat. Obšč. 2 (1953), 201-233 (Russian); MR 15, 442.

82. G. I. Silova, Existence of an absolute minimum of multiple integrals of the calculus of variations, Dokl. Akad. Nauk SSSR (N.S.) 102 (1955), 699-702; MR 17, 46.

83. S. Sobolev, On a theorem of functional analysis, Mat. Sb. (N.S.) 4 (1938), 471-497.

84. G. Stampacchia, Contributi alla regolarizzazione delle soluzioni dei problemi al contorno per equazioni del secondo ordine ellitiche, Ann. Scuola Norm. Sup. Pisa (III) 12 (1958), 223-244.

85. - Problemi al contorno ellittici, con dati discontinui, dotati di soluzioni hölderiane, Ann. Mat. Pura Appl. (IV) 51 (1960), 1-38.

86. L. Tonelli, Fondamenti del calcolo delle variazioni, Bologna, Zanichelli, 3 vols.

87. - Sur la semi-continuité des intégrales doubles du calcul des variations, Acta Math. 53 (1929), 325-346.

88. - L'estremo assoluto degli integrali doppi, Ann. Scuola Norm. Pisa (II) 3 (1933), 89-130.

89. L. Van Hove, Sur l'extension de la condition de Legendre du calcul des variations aux intégrals multiples a plusieurs fonctions inconnues, Nederl. Akad. Wetensch. $\mathbf{5 0}$ (1947), 18-23.

90. M. I. Visik, On strongly elliptic systems of differential equations, Mat. Sb. (N.S.) 29(71) (1951), 615-676.

91. A. Weinstein, Etude des spectres des équations aux dérivées partielles de la théorie des plaques élastiques, Mem. Sci. Math., Fasc. 88, Gauthier-Villars, Paris, 1937.

92. L. C. Young, On generalized surfaces of finite topological type, Mem. Amer. Math. Soc. No. 17 (1955).

93. K. Yosida, Lectures on semi-group theory and its application to Cauchy's problem in partial differential equations, Tata Institute Lectures on Mathematics and Physics Math. 8.

\section{ADDITIONAL BIBLIOGRAPHY}

\section{Books}

F. John, Plane waves and spherical means applied to partial differential equations, Interscience, New York, 1955.

S. Bergman, Kernel functions and elliptic differential equations in mathematical physics, Academic Press, New York, 1953.

C. Carathéodory, Variationsrechnung und partielle Differentialgleichungen erster Ordnung, Band I, 2te Aufl., B. G. Teubner, Leipzig, 1956.

\section{Sets of Notes}

J. L. Lions, Lectures on elliptic partial differential equations, Tata Institute of Fundamental Research, Lectures on Mathematics, No. 10. 
Kans.

, Boundary value problems, Dept. of Math., Univ. of Kansas, Lawrence,

L. Nirenberg, Existence theorems in partial differential equations, New York University, Inst. Math. Sci.

L. Schwartz, Lectures on mixed problems in partial differential equations and representations of semigroups, Tata Institute of Fundamental Research, 1957.

\section{Proceedings of certain symposia}

Contributions to the theory of partial differential equations, Ann. of Math. Studies No. 33, Princeton Univ. Press, Princeton, N. J., 1954.

Proc. of the 1955 Berkeley Symposium

(a) Lectures, Dept. of Math., Univ. of Kansas, Lawrence, Kans.

(b) Transactions, Comm. Pure Appl. Math. 9 (1956).

Proc. Conference on Partial Differential Equations, Univ. of Kansas, Lawrence, Kans., 1954.

Partial differential equations, Proc. Sympos. Pure Math. Vol. 4, American Mathematical Society, Providence, R. I., 1962 (1960 Berkeley Symposium).

Partial differential equations and continuum mechanics, Math. Res. Center, Univ. of Wisconsin Press, Madison, Wis., 1961.

\section{Expository articles}

L. Gårding, Some trends and problems in linear differential equations, Proc. Int. Congr. Math., 1958.

G. Stampacchia, I problemi al contorno per le equazioni differenziali di tipo ellittico, Atti VI Congr. Un. Mat. Ital. (Naples, 1959), pp. 21-44, Edizione Cremonese, Rome 1960.

A. Weinstein, Bounds for eigenvalues and the method of intermediate problems, Partial Differential Equations and Continuum Mechanics, pp. 39-53, Math. Res. Center, Univ. of Wisconsin Press, Madison, Wis., 1961.

The University of California, Berkeley 